\title{
Connecting neutron star observations to the high density equation of state of quasi-particle model
}

\author{
Yan Yan ${ }^{1}$, Jing $\mathrm{Cao}^{1}$, Xin-Lian $\mathrm{Luo}^{2}$, Wei-Min Sun ${ }^{1,3}$ and Hongshi Zong ${ }^{1,3 *}$ \\ ${ }^{1}$ Department of Physics, Nanjing University, Nanjing, 210093, China \\ ${ }^{2}$ Department of Astronomy, Nanjing University, Nanjing 210093, China and \\ 3 Joint Center for Particle, Nuclear Physics and Cosmology, Nanjing 210093, China
}

\begin{abstract}
The observation of $1.97 \pm 0.04$ solar-mass neutron-like star gives constraint on the equation of state (EOS) of cold, condensed matter. In this paper, the EOS for both pure quark star and hybrid star with a quark core described by quasi-particle model are considered. The parameters of quasiparticle model which affect the mass of both quark star and hybrid star can be constrained by the observation.

PACS numbers: 12.38.AW, 12.39.Ba, 14.65.Bt, 97.60.Jd
\end{abstract}

\section{INTRODUCTION}

It is well known that the equation of state (EOS) for cold and dense strongly interacting matter plays a key role in the study of the neutron stars in astrophysics [1, 2]. The neutron star are presumed to contain the densest matter in the cosmos, which provide a natural laboratory for cold, condensed matter. The recent observation [3] of the pulsar PSR J1614-2230 with a mass of $1.97 \pm 0.04 M_{\odot}\left(M_{\odot}\right.$ denotes the mass of the sun) gives a strong constraint on the EOS of strongly interacting matter.

Just as the discovery of neutron led to the idea of neutron star, the conception of quark naturally stimulated many physicists and astrophysicists to suggest that neutron-like star may be quark or hybrid star (Quark or hybrid star is composed, in whole or in part, of quark matter) [4 9]. At present, it is still not possible to get a reliable EOS of quark matter from the first principle of Quantum Chromodynamics (QCD). In this case, a commonly used approach in the study of high density quark matter is to give up on first principle calculations and resort to phenomenological QCD models, such as MIT bag model [10-13], NJL model [14 18], perturbative QCD model [19 22] and quasi-particle model 23 26]. Among these models, the quasi-particle quarkgluon plasma model with a few fitting parameters, has been widely used to reproduce the properties of the QCD plasma at finite temperature and zero chemical potential [12, 27 33]. However, due to lack of experimental data on dense strongly interacting matter at zero temperature and finite chemical potential, the phenomenological parameters of quasi-particle model at zero temperature and finite chemical potential has some uncertainties. The main purpose of this paper is try to constrain the parameters of the quasi-particle model at finite chemical potential based on the most recent astronomical observation [3].

*Email:zonghs@chenwang.nju.edu.cn
This paper is organized as follows. In Section II, we introduce the quasi-particle model and its parameters. In Section III, the structure of neutron-like star is discussed. The relation between the parameters of quasiparticle model and mass-radii relation of neutron-like star is also illustrated. Through comparison of theoretical results with astrophysical observation [3], one can give a strong constraint on the parameters of quasi-particle model used in the present work. Finally, a summary is given in Section IV.

\section{THE QUASI-PARTICLE MODEL AND ITS PARAMETERS}

Recently, by means of functional integral formalism a model-independent formula for calculating the EOS of QCD at finite chemical potential and zero temperature was proposed in Ref. [34]. By using this formula the authors in Ref. [35] obtained an explicit analytic expression for the EOS using the quasi-particle model. In this paper, we shall use the EOS of this model to study the structure of the neutron-like star. The EOS in Ref. 35] is obtained by using path integral method with a effective quark propagator. This quark propagator has the form of a free quark propagator with a density dependent effective mass.

According to Ref. [34], the quark number density reads

$$
\rho(\mu)=-N_{c} N_{f} \int \frac{d^{4} p}{(2 \pi)^{4}} \operatorname{tr}\left\{G[\mu](p) \gamma_{4}\right\}
$$

where $G[\mu](p)$ is the full quark propagator at finite chemical potential $\mu, N_{c}$ and $N_{f}$ denote the number of colors and of flavors, respectively, and the trace operation is over Dirac indices. According to the above formula, in order to calculate the quark number density, one should know the full quark propagator at finite $\mu$ in advance. Unfortunately, at present no one knows how to calculate the full quark propagator from first principles of QCD. So, one has to choose some effective model quark propagator as the approximation of the full quark propagator. 
In this paper we choose the following model quark propagator proposed in Ref. [35]

$$
G^{-1}[\mu](p)=i \gamma \cdot \widetilde{p}+m(\mu),
$$

where $\widetilde{p} \equiv\left(\vec{p}, p_{4}+i \mu\right)$ and the Euclidean gamma matrices satisfy the algebra $\left\{\gamma_{\mu}, \gamma_{\nu}\right\}=2 \delta_{\mu \nu}$. Here, following Refs. [36, 37], we choose the effective quark mass $m(\mu)$ and the effective coupling constant $g^{2}(\mu)$ to be:

$$
m^{2}(\mu)=\frac{\mu^{2} g^{2}(\mu)}{3 \pi^{2}}, \quad g^{2}(\mu)=\frac{16 \pi^{2}}{9 \ln (a(\mu+c))^{2}},
$$

where $a, c$ are phenomenological parameters of quasiparticle model. In this work, the current mass of $\mathrm{u}, \mathrm{d}, \mathrm{s}$ quarks is taken to be zero. It could satisfy chemical equilibrium and neutrality naturally. Then we can use the contour integration method to obtain the quark number density

$$
\rho(\mu)=\frac{N_{c} N_{f}}{3 \pi^{2}}\left(\mu^{2}-m^{2}(\mu)\right)^{3 / 2} \theta(\mu-m(\mu)) .
$$

Due to the step function in the right-hand side of Eq. (4), it can be found that the quark number density vanishes when $\mu$ is below a critical value $\mu_{0}$ (In this work, the value of $\mu_{0}$ depends on the parameters $a$ and $c$ ). Namely, $\mu=\mu_{0}$ is a singularity which separates two regions with different quark number densities. This behavior agrees qualitatively with the general conclusion of Ref. [38]. Now let us turn to the calculation of the EOS. According to Refs. [39, 40], the EOS of QCD at $T=0$ reads

$$
P(\mu)=\left.P(\mu)\right|_{\mu=0}+\int_{0}^{\mu} d \mu^{\prime} \rho\left(\mu^{\prime}\right)
$$

where $\left.P(\mu)\right|_{\mu=0}$ is vacuum pressure which represents the pressure density at $\mu=0$. In this paper, we take $\left.P(\mu)\right|_{\mu=0} \equiv-B$, where $B$ is a phenomenological parameter in our present work (at present one cannot calculate its numerical value reliably from first principles of QCD). Here it should be noted that Eq. (5) is a model independent formula. This formula shows that the pressure density $P(\mu)$ at finite $\mu$ and zero temperature is totally determined by the quark number density $\rho(\mu)$ (up to a constant term $\left.\left.P(\mu)\right|_{\mu=0}\right)$. Therefore, the quark number density has the correct behavior required by QCD implies that the pressure $P(\mu)$ also has the correct behavior required by $\mathrm{QCD}$ at finite chemical potential and zero temperature.

Substituting Eq. (4) into Eq. (5), we obtain

$P(\mu)=-B+\frac{3}{\pi^{2}} \int_{0}^{\mu} d \mu^{\prime} \theta\left(\mu^{\prime}-m\left(\mu^{\prime}\right)\right)\left(\mu^{\prime 2}-m^{2}\left(\mu^{\prime}\right)\right)^{3 / 2}$,

where we have taken $N_{f}=N_{c}=3$. From Eq. (6) it can be seen that the EOS of quasi-particle model depends on the model parameters $a, c$. Moreover, the so-called vacuum pressure $B$ is also a parameter which affects the

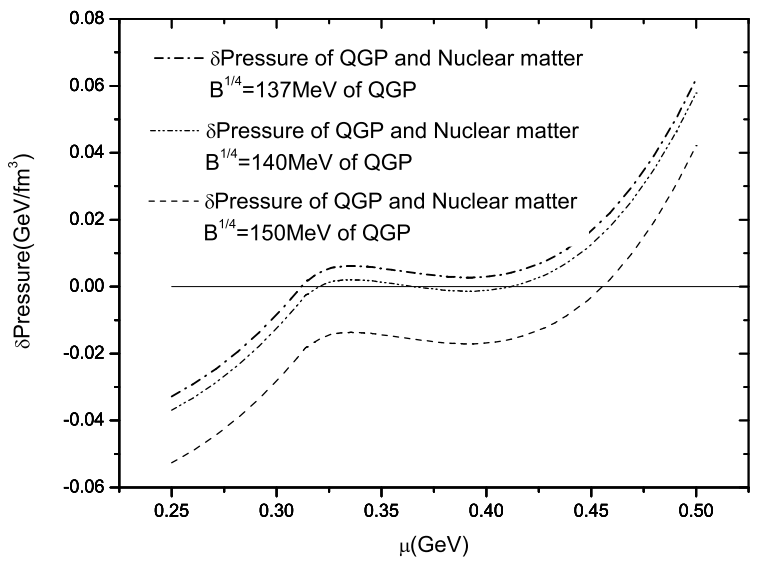

Fig. 1. The $\delta$ Pressure of quark matter described by quasi-particle model $(a=12 / \mathrm{GeV}, c=-0.05 \mathrm{GeV})$ and hadronic matter with different $B$

EOS in the study of neutron-like star [41 43]. In the following, we shall discuss the relation between parameters $a, c$ and $B$.

The choice of $B$ in quasi-particle model is complicated (depending on the corresponding hadronic EOS [44] and quark EOS). The different parameters $a, c$ in quasi-particle model lead to different ranges of $B$. In order to see it clearly, let us to define $\delta P(\mu) \equiv P_{\text {quark }}(\mu)-$ $P_{\text {hadron }}(\mu)$ to study the phase transition. Here $P_{\text {quark }}(\mu)$ and $P_{\text {hadron }}(\mu)$ denote the pressure of quark matter and hadronic matter, respectively. $P_{\text {quark }}(\mu)$ can be obtained using Eq. (6). According to the EOS in Ref. [44], $P_{\text {hadron }}=0$ when $\mu<307 \mathrm{MeV}$. A physical phase transition infers that $\delta P(\mu)$ intersects with 0 once when $\mu>307 \mathrm{MeV}$, that is $B>\left.P_{\text {quark }}(0.307 \mathrm{GeV})\right|_{\mu \neq 0}$, where $\left.P_{\text {quark }}(\mu)\right|_{\mu \neq 0}$ stands for the second term in the righthand side of Eq. (6). However, the case is not always so simple. We show $\delta P(\mu)$ in Fig. 1 with model parameters $a=12 / \mathrm{GeV}, c=-0.05 \mathrm{GeV}$. Just as is shown in Fig. 1, $\delta P(\mu)$ is not even a monotonously increasing function. There are two peaks $\delta P\left(\mu_{1}\right)$ and $\delta P\left(\mu_{2}\right)$, $\mu_{1}<\mu_{2}$. We have two choices to get a valid result: (1) $\delta P\left(\mu_{1}\right)<0$, that is $B>\left.P_{\text {quark }}\left(\mu_{1}\right)\right|_{\mu \neq 0}-P_{\text {hadron }}\left(\mu_{1}\right)$, i.e., $B^{1 / 4}=150 \mathrm{MeV}$ in Fig. 1 ; $(2) \delta P\left(\mu_{2}\right)>0$ and $B>P_{\text {quark }}(0.307 \mathrm{GeV})$, that is $\left.P_{\text {quark }}(0.307 \mathrm{GeV})\right|_{\mu \neq 0}<$ $B<\left.P_{\text {quark }}\left(\mu_{2}\right)\right|_{\mu \neq 0}-P_{\text {hadron }}\left(\mu_{2}\right)$, i.e., $B^{1 / 4}=137 \mathrm{MeV}$ in Fig. 1. It can be seen that some values of $B$ (i.e., $B^{1 / 4}=140 \mathrm{MeV}$ ) would lead to the result which is not physical. It may be due to the inconsistency of QGP model and constant vacuum pressure. At present we do not have a valid method to deal with it, instead, when we use QGP model to study the structure of the neutronlike star, we should pay attention to the range of $B$. For some sets of model parameters $a, c$, the second choice does not exist because $\left.P_{\text {quark }}\left(\mu_{2}\right)\right|_{\mu \neq 0}-P_{\text {hadron }}\left(\mu_{2}\right)<$ $\left.P_{\text {quark }}(0.307 \mathrm{GeV})\right|_{\mu \neq 0}$.

The mass-radii relation of neutron-like star depends 


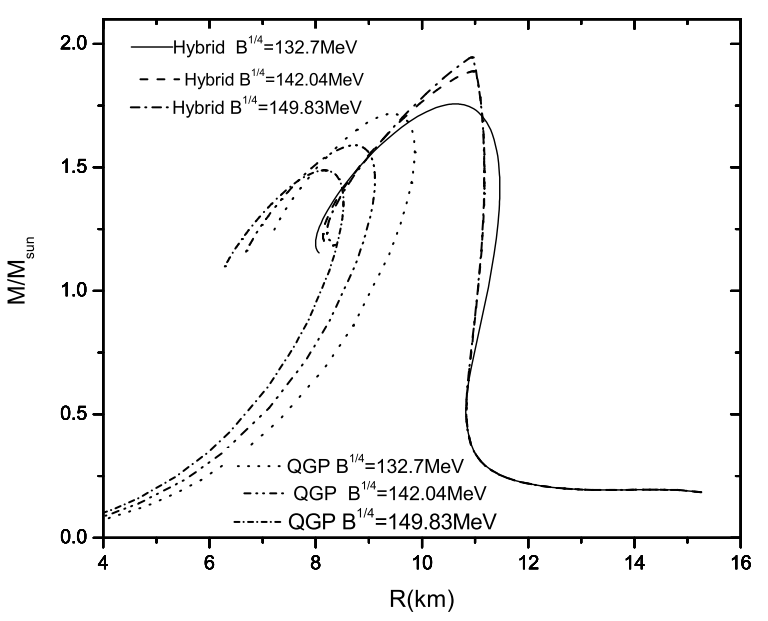

Fig. 2. The mass-radii relations of hybrid star and pure quark star with $a=13 / \mathrm{GeV}, \mathrm{c}=-0.1 \mathrm{GeV}$ and different $B$

obviously on $B$. The mass-radii relation of the pure quark star and the hybrid star (with $a=13 / \mathrm{GeV}, c=$ $-0.1 G e V$ ) with different $B$ are plotted in Fig. 2 . From Fig. 2 it can be seen that for pure quark star lager $B$ yields smaller mass and radius, while the case for hybrid star is more complicated. We will discuss it in Part B of Section III.

\section{THE STRUCTURE OF NEUTRON-LIKE STAR}

Using the EOS, we can obtain the structure of a neutron-like star by integrating the TolmanOppenheimer-Volkoff equation

$$
\begin{aligned}
& \frac{d P(r)}{d r}=-\frac{G(\varepsilon+P)\left(M+4 \pi r^{3} P\right)}{r(r-2 G M)}, \\
& \frac{d M(r)}{d r}=4 \pi r^{2} \varepsilon .
\end{aligned}
$$

The energy density reads

$$
\varepsilon(\mu)=-P(\mu)+\mu \cdot \frac{\partial P}{\partial \mu} .
$$

In this paper, we consider the interior structure of a neutron-like star composed, in whole or in part, of quark matter. The structure of pure quark star is described totally by the quasi-particle model. The hybrid star which is proposed by the authors of Refs. [7 9] has a quark core described by quasi-particle model and a crust of nuclear matter described by the APR hadronic EOS [44].

\section{A. Pure Quark Star}

In this part, we use the recent observation of $1.97 \pm$ 0.04-solar-mass neutron-like star [3] to constrain the pa-

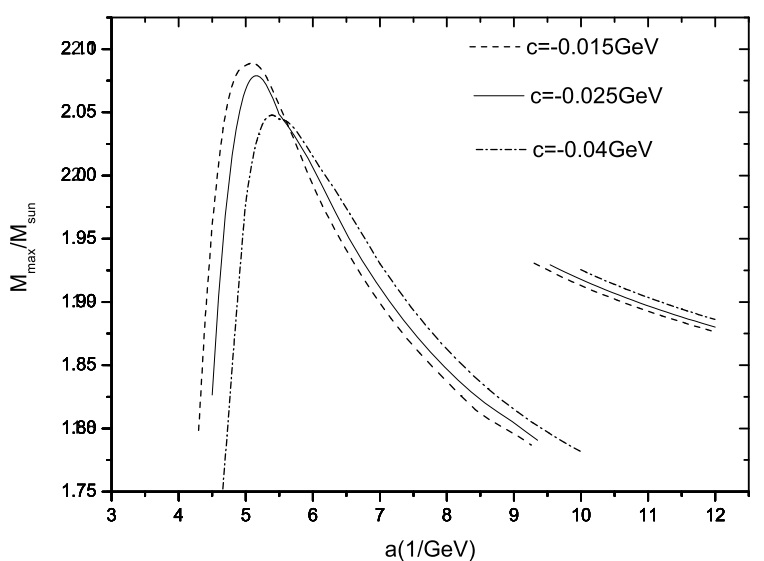

Fig. 3.The maximal mass changes with the parameter $a$ for different $c$.

rameters $a, c$. In the previous section, we show that the larger $B$ yields smaller mass, so we can choose the smallest $B$ which leads to the valid result to get the maximal mass for every set of $a, c$.

The relation between the mass and model parameters $a, c$ is shown in Fig. 3. It can be seen clearly that for the same $c$, the mass for different $a$ increases at first, then decreases later. This is because at first the smallest $B \approx 0$, larger $a$ yields larger mass. When $B$ dominates the maximal mass, larger $a$ leads to larger $B$ which yields smaller mass. This two obvious regions come from the different choices of $B$ which we discuss in Section II. For different $c$, larger $c$ yields larger mass at first, then the larger $c$ leads to smaller mass. The largest maximal mass to $a$ increases with $c$ increasing.

With the mass related to the the parameters $a, c$, we can constrain the parameters with the recent observation of 1.97 \pm 0.04 -solar-mass neutron-like star [3]. We assume it is a pure quark star which can be described by quasiparticle model.

The range of $a, c$ is illustrated in Fig. 4. The physical meaning of the parameters $a, c$ is not obvious. Here, we try to understand it from the point of view of the running coupling constant $\alpha_{s}$. The comparison of the experimental data on $\alpha_{s}$ and the results of $\alpha_{s}$ calculated with some representative values of $a, c$ are shown in Fig. 5. Here, one point should be clarified. In the usual expression of the running coupling constant $\alpha_{s}\left(Q^{2}\right), \alpha_{s}$ is a function of the momentum scale $Q$. In our work, in order to understand the physical meaning of $\alpha_{s}$, the chemical potential $\mu$ is taken as an energy scale similar to the momentum scale $Q$ in the expression of $\alpha_{s}$. From Fig. 5 and Fig. 6 (see below), it can be seen that the parameters which yield $1.97 \pm 0.04 M_{\odot}$ are also consistent with the experimental data on $\alpha_{s}$ [45]. Therefore, we conclude that quasi-particle model with proper parameters is suitable to describe quark matter.

If we assume the central density $\mu$ of quark star to be 


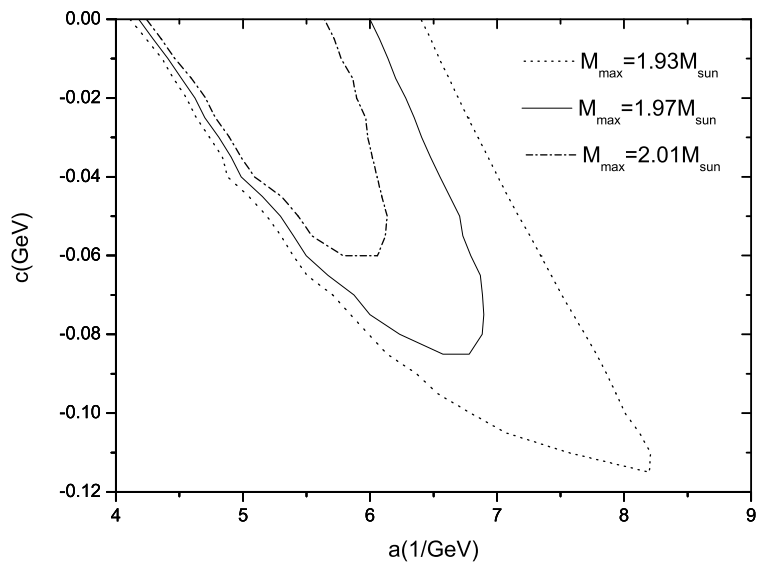

Fig. 4. The contour of the parameters $a, c$ constrained by recent observation [3]

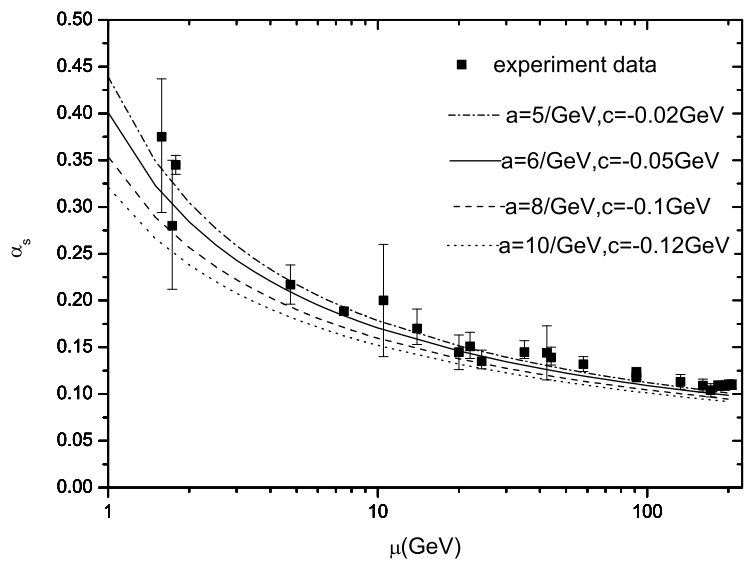

Fig. 5. Comparison of the running coupling constant $\alpha_{s}$ calculated using different values of $a, c$ with the experimental data. The experimental data are taken from table 1 of Ref. [45].

$0.45 \mathrm{GeV}$, from Fig. 6 we deduce it is reasonable, then the range of $\alpha_{s}$ is from $0.659 \sim 1.135$. By calculation we can also find that the range of $\mu_{0}$ is $0.21 \mathrm{GeV} \sim 0.327 \mathrm{GeV}$. In Ref. [38], based on a universal argument, it is pointed out that the existence of some singularity at the point $\mu=\mu_{0}$ and $T=0$ is a robust and model-independent prediction. The numerical value of critical chemical potential in pure QCD (i.e., with electromagnetic interactions being switched off) is estimated to be $\frac{m_{N}-16 \mathrm{MeV}}{N_{c}}=307 \mathrm{MeV}$ (where $m_{N}$ is the nucleon mass and $N_{c}=3$ is the number of colors). Here, in order to be self-contained, we shall give an explanation for how this result is derived. According to the argument of Ref. [38], the critical baryon chemical potential $\mu_{B 0}=\min _{\alpha}\left(E_{\alpha} / N_{\alpha}\right)$, where $\alpha$ stands for a generic quantum state of the system and $E_{\alpha}$ and $N_{\alpha}$ is the energy and baryon number of this state, respectively. The energy per baryon, $E / N$, can

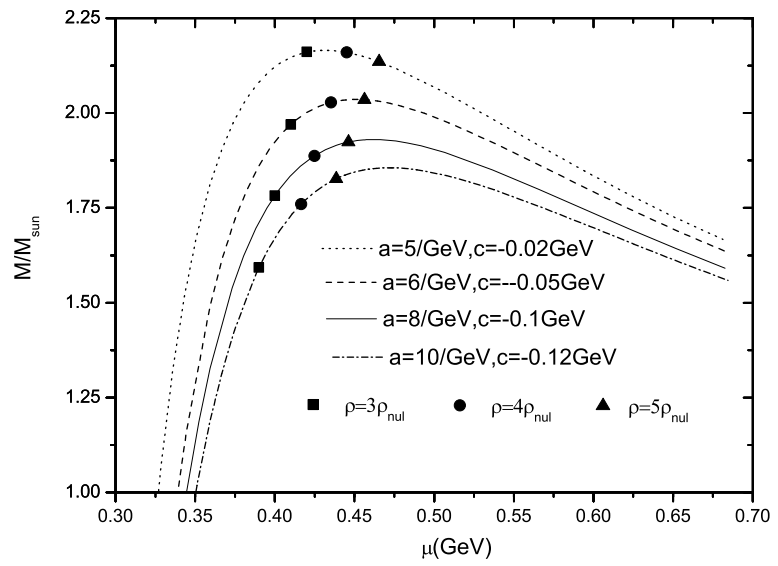

Fig. 6. Mass of different central potential with different parameters $a, c$, where $\rho_{\text {nul }}=2.8 \times 10^{14} \mathrm{~g} / \mathrm{cm}^{3}$. $B$ is chosen to be smallest which is physical to obtain the maximal mass.

also be written as $m_{N}-\left(N m_{N}-E\right) / N$, where $m_{N}$ is the nucleon mass. Therefore, the state which minimizes $E / N$ is that for which the binding energy per nucleon, $\epsilon=\left(N m_{N}-E\right) / N$, is a maximum. Empirically, we know that this state is a single iron nucleus at rest with $N=A=56$ and $\epsilon \approx 8 \mathrm{MeV}$. However, in QCD without electromagnetism the binding energy per nucleon increases with $N=A$. This is the consequence of the saturation of nuclear forces and can be seen from the Weizsacker formula. Without electromagnetism, only the bulk and surface energy terms are significant for large $A$ :

$$
\epsilon(A) \equiv \frac{A m_{N}-m_{A}}{A} \approx a_{1}-a_{2} A^{-1 / 3}
$$

with $a_{1} \approx 16 \mathrm{MeV}, a_{2} \approx 18 \mathrm{MeV}$ [46]. As $A \rightarrow \infty$, $\epsilon$ saturates at the value $a_{1}$. This corresponds to the binding energy per nucleon in a macroscopically large sample of nuclear matter as defined by Fetter and Walecka in [46]. Therefore, in QCD the critical baryon chemical potential $\mu_{B 0} \approx m_{N}-16 \mathrm{MeV}$ and the corresponding quark chemical potential $\mu_{0}$ is $\frac{m_{N}-16 \mathrm{MeV}}{N_{c}}=307 \mathrm{MeV}$. The range of parameters $a, c$ constrained by the observation of $1.97 \pm 0.04$-solar-mass neutron-like star [3] is consistent with it.

\section{B. Hybrid Star}

The hybrid star was inspired by the idea of the phase transition from quark matter to nuclear matter [7-9, 47]. The phase transition in this paper is modeled by a simple Maxwell's construction.

In Fig. 7 we plot the relation of the maximal mass to the parameter $B$. From Fig. 7 it can be seen that in the large $B$ region, the maximal mass tends to $2.2 M_{\odot}$. This means that for large $B$, the hybrid star tends to a star which is composed totally by hadronic matter. 


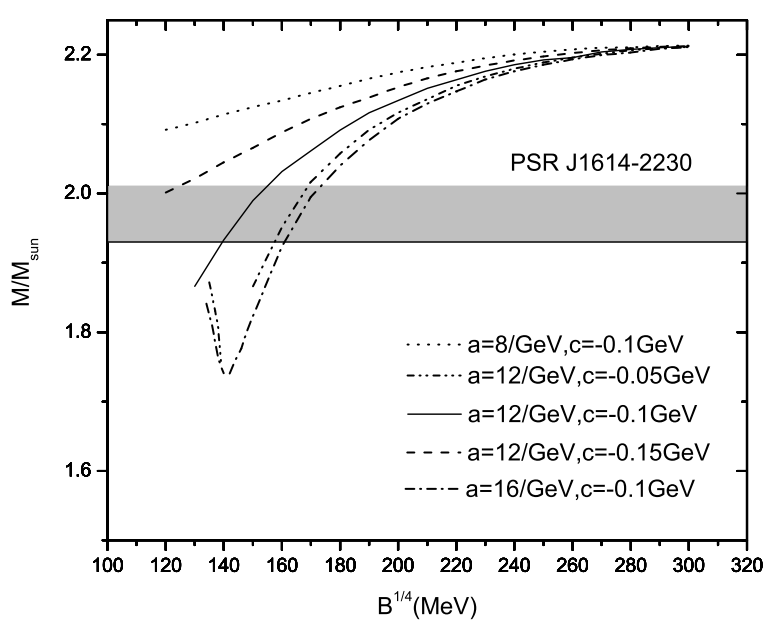

Fig. 7. The maximal mass changes with the parameter $B$ with different $a, c$.

The maximal mass for small $B$ depends on the parameters in quasi-particle model. For some parameters (e.g., $a=12 / \mathrm{GeV}, c=-0.1 \mathrm{GeV})$, the maximal mass increases with $B$ increasing, while for other parameters (e.g., $a=12 / \mathrm{GeV}, c=-0.05 \mathrm{GeV}$ ), the maximal mass decreases with $B$ increasing at the beginning, then it increases with $B$ increasing. This means that for the latter parameters, the behavior at small $B$ tends to a pure quark star. The discontinuous curves with parameters $a=12 / \mathrm{GeV}, c=-0.05 \mathrm{GeV}$ and $a=16 / \mathrm{GeV}, c=$ $-0.1 \mathrm{GeV}$ shown in Fig. 7 is caused by the valid ranges of $B$. We can compare the result with the same plot published in Ref. [48] which has a quark core described by MIT bag model and hadronic crust described by TM1 RMF EOS and NL3 RMF EOS. However, the running coupling constant in the center of the star in Fig. 7 is narrow. In Fig. 8, we plot the maximal mass to $B$ with different central coupling constant. The plot is similar with the result in Ref. [48]. In Ref. [48], the authors stressed that different hadronic model could lead to the differences of the result. In our work, we use APR EOS to describe the hadronic crust.

From Fig. 9 and Fig. 11, a smaller $B$ will narrow the region of $a-c$ space. However, for hybrid star we cannot give a rigid range of $a$ and $c$. It can also be figured out that smaller $a$ and $c$ will yield larger maximal mass. According to Eq. (3), larger running coupling constant leads to larger mass, which is consistent with the $1.97 \pm$ 0.04 solar-mass neutron-like star.

\section{SUMMARY}

The parameters of quasi-particle model at zero temperature and finite chemical potential can be constrained by recent observation [3]. We consider the structure of a neutron star which is composed, in whole or in part, of

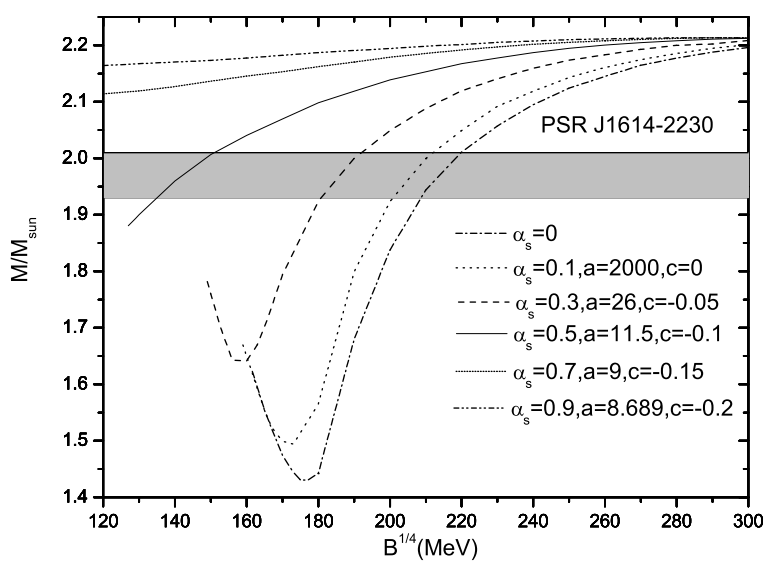

Fig. 8. The maximal mass changes with the parameter $B$ with different $\alpha_{s}$.

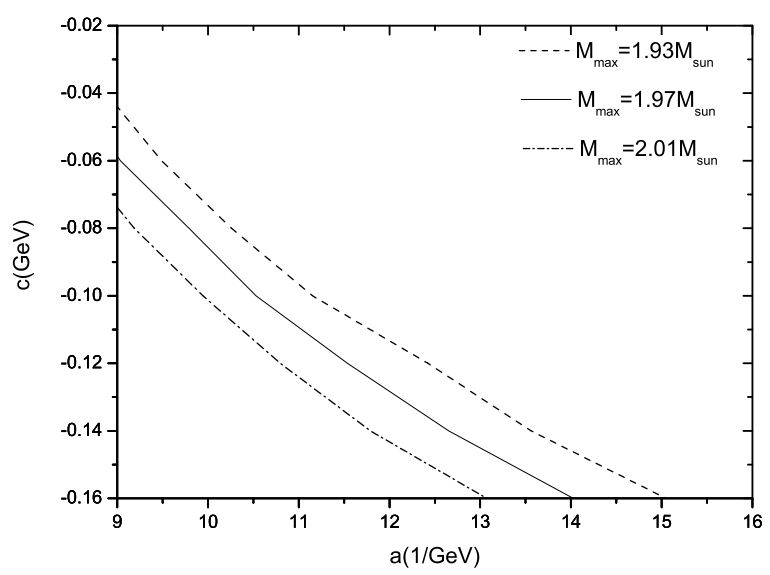

Fig. 9. The contour picture of parameters in $B^{1 / 4}=130.58 \mathrm{MeV}$

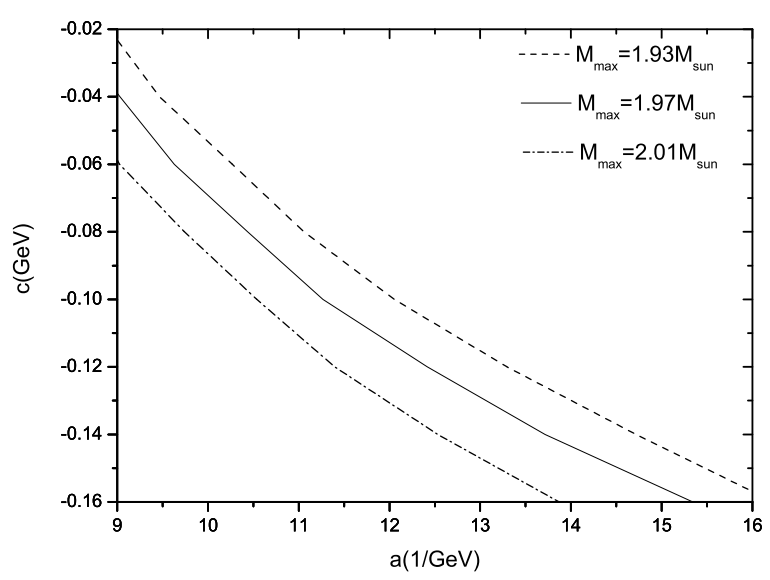

Fig. 10. The contour picture of parameters in $B^{1 / 4}=140.32 \mathrm{MeV}$ 


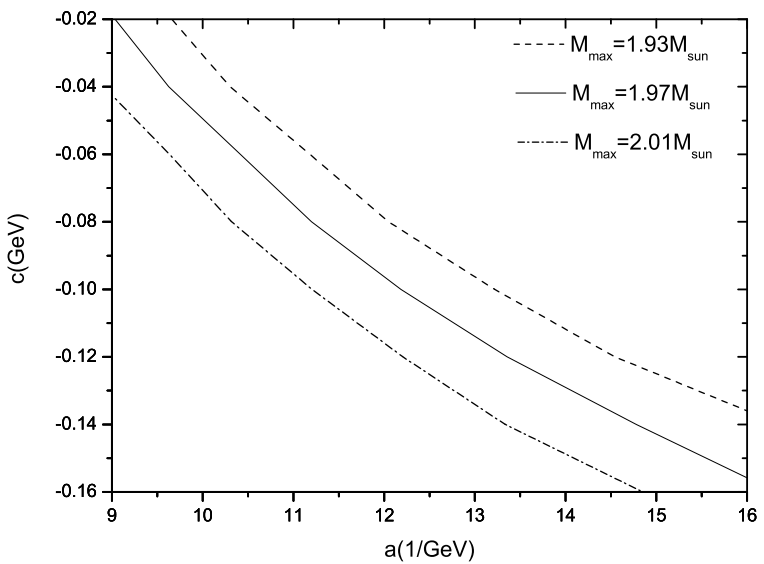

Fig. 11. The contour picture of parameters in $B^{1 / 4}=148.37 \mathrm{MeV}$

quark matter. In this paper quark matter is described by quasi-particle model incorporating a bag constant $B$.

We discuss the range of $B$ and the influences of $B$ on both quark and hybrid star. First, we assume the neutron-like star is a pure quark star. Larger $B$ yields smaller mass. We can constrain the parameters $a, c$ by recent observation [3]. The range of the running coupling constant $\alpha_{s}$ with central density $\mu=0.45 \mathrm{GeV}$ of pure quark star is $0.659 \sim 1.135$ (this clearly shows that even at such a high density the coupling constant is still large, so perturbation theory cannot be used). We can also find that the range of $\mu_{0}$ is $0.21 \mathrm{GeV} \sim 0.327 \mathrm{GeV}$, which is consistent with the result given in Ref. [38]. Second, we assume the neutron-like star to be a hybrid star which has a quark core and a hadronic crust. The parameters of quasi-particle model and the vacuum pressure $B$ affect the structure of hybrid star. In the large $B$ region, the maximal mass tends to $2.2 M_{\odot}$. The maximal mass for small $B$ depends on the parameters of quasi-particle model. For some parameters (e.g., $a=12 / \mathrm{GeV}, c=-0.1 \mathrm{GeV})$, the maximal mass increases with $B$ increasing, while for other parameters (e.g., $a=12 / \mathrm{GeV}, c=-0.05 \mathrm{GeV}$ ), the maximal mass decreases with $B$ increasing at the beginning, then it increases with $B$ increasing. Larger $a$ and $c$ lead to smaller mass of hybrid star. We conclude that either a pure quark star and a hybrid star with a quark core described by quasi-particle model can be consistent with the recent observation of PSR J1614-2230. The parameters in quasi-particle model can be narrowed by the observation.

This work is supported in part by the National Natural Science Foundation of China (under Grant Nos 10775069, 10935001 and 11075075) and the Research Fund for the Doctoral Program of Higher Education (under Grant No 200802840009)
[1] F. Özel, Nature 441, 1115 (2006).

[2] M. Alford et al., Nature 445, E7 (2007).

[3] P.B. Demorest, T. Pennucci, S.M. Ransom, M.S.E. Roberts, and J.W.T. Hessels, Nature 467, 1081-1083 (2010).

[4] G. Zweig, CERN Report No. TH-401 (1964).

[5] G. Zweig, CERN Report No. TH-412 (1964).

[6] N. Itoh, Prog, Theor. Phys. 44, 291 (1970).

[7] K. Schertler, C. Greiner, P.K. Sahu, and M.H. Thoma, Nucl. Phys. A. 637, 451 (1998).

[8] K. Schertler, C. Greiner, J. Schaffner-Bielich, and M.H. Thoma, Nucl. Phys. A. 677, 463 (2000).

[9] N.K. Glendenning, Phys. Rep. 342, 393 (2001).

[10] A. Chodos, R.L. Jaffe, K. Johnson, C.B. Thorn and V.F. Weisskopf, Phys. Rev. D 9, 3471 (1974).

[11] F. Weber, Pulse as Astrophysical Laboratories for $\mathrm{Nu}$ clear and Particle Physics (Iop, Bristal, 1999).

[12] A. Peshier, B. Kampfer, and G. Soff, Phys. Rev. C 61, 045203 (2000).

[13] M. Alford, M. Braby, M. Paris, and S. Reddy, Astrophys. J. 629, 969 (2005).

[14] P. Rehberg, S.P. Klevansky, and J. Hüfner, Phys. Rev. C 53, 410(1996).

[15] M. Hanauske, L.M. Satarov, I.N. Mishustin, H. Stocker, and W. Greiner, Phys. Rev. D 64, 043005 (2001).

[16] S.B. Rüster, D.H. Rischke, Phys. Rev. D 69, 045011 (2004).

[17] D.P. Menezes, C. Providencia, D.B. Melrose, J. Phys. G:
Nucl. Part. Phys, 32,1081 (2006).

[18] Y. Jiang, H. Gong, W.M. Sun, and H.S. Zong, Phys. Rev. D 85, 034031 (2012).

[19] B. Freedman and L.McLerran, Phys. Rev. D 16, 1130 (1977); ibid. 16, 1147 (1977); ibid. 16, 1169 (1977); ibid. 17, 1109 (1978).

[20] V. Baluni, Phys. Rev. D 17, 2092 (1978).

[21] E.S. Fraga, R.D. Pisarski and J. Schaffner-Bielich, Phys. Rev. D 63, 121702 (2001).

[22] E. Farhi and R.L. Jaffe, Phys. Rev. D 30, 2379 (1984).

[23] V. Goloviznin, H. Satz, Z. Phys. C 57, 671 (1993).

[24] A. Peshier, B. Kampfer, O.P. Pavlenko, and G. Soft, Phys. Lett. B 337, 235 (1994).

[25] M.I. Gorenstein and S.N. Yang, Phys. Rev. D 52, 5206 (1995).

[26] J. Cao, Y. Jiang, W.M. Sun, and H.S. Zong, Phys. Lett. B 771, 65 (2012).

[27] P. Levai, U. Heinz, Phys. Rev. C 57, 1879 (1998).

[28] R.A. Schneider and W. Weise, Phys. Rev. C 64, 055201 (2001).

[29] K.K. Szabó and A.I. Tóth, JHEP 06, 008 (2003).

[30] M.A. Thaler, R.A. Schneider, and W. Weise, Phys. Rev. C 69, 035210 (2004).

[31] M. Bluhm, B. Kämpfer, and G. Soff, Phys. Lett. B 620, 131 (2005); M. Bluhm, and B. Kämpfer, Phys. Rev. D 77, 034004 (2008).

[32] V.M. Bannur, Eur. Phys. J. C 50, 629 (2007); ibid, Phys. Lett. B 647, 271 (2007); ibid, Phys. Rev. C 75, 044905 
(2007); ibid, JHEP 09, 046 (2007); ibid, Phys. Rev. C 78, 045206 (2008).

[33] S. Plumari, W.M. Alberico et al., Phys. Rev. D 84, 094004 (2011).

[34] H.S. Zong and W.M. Sun, Int. J. Mod. Phys. A 23, 3591 (2008).

[35] A.M. Zhao, J. Cao, L.J. Luo, W.M. Sun, and H.S. Zong, Mod. Phys. Lett. A 25, 47 (2010).

[36] X.P. Zhang, M. Kang, X.W. Liu, and S.H. Yang, Phys. Rev. C 72, 025809 (2005).

[37] M. Bluhm and B. Kämpfer, Phys. Rev. D 77, 034004 (2008).

[38] M.A. Halasz, A.D. Jackson, R.E. Shrock, M.A. Stephanov, and J.J.M. Verbaarschot, Phys. Rev. D 58, 096007 (1998).

[39] M. He, W.M. Sun, H.T. Feng, and H.S. Zong, J. Phys. G 34, 2655 (2007).

[40] H.S. Zong and W.M. Sun, Phys. Rev. D 78, 054001
(2008).

[41] H. Li, X.L. Luo, and H.S. Zong, Phys. Rev. D 82, 065017 (2010).

[42] H. Li, X.L. Luo, Y. Jiang, and H.S. Zong, Phys. Rev. D 83, 025012 (2011).

[43] Y.L. Tian, Y. Yan, H. Li, X.L. Luo, and H.S. Zong, Phys. Rev. D 85, 045009 (2012).

[44] A. Akmal, V. R. Pandharipande, and D. G. Ravenhall, Phys. Rev. C 58, 1804 (1998).

[45] S. Bethke, arXiv: 0606035 [hep-ex].

[46] A.L. Fetter and J.D. Walecka, Quantum Theory of ManyParticle Systems (McGraw-Hill, New York, 1971).

[47] A. Kurkela, P. Romatschke, A. Vuorinen, and B. Wu, arXiv: 1006.4062 [astro-ph.HE].

[48] S. Weissenborn, I. Sagert, G. Pagliara, M. Hempel, J. Schaffner-Bielich, arXiv: 1102.2869 [astro-ph.HE]. 